\title{
Glucose induced biofilm formation in Bacillus thuringiensis KPWP1 is associated with increased cell surface hydrophobicity and increased production of exopolymeric substances
}

\section{Sushmita Jha}

Indian Institute of Science Education and Research Kolkata

Nirbhay Bhadani

Indian Institute of Science Education and Research Kolkata

Abhinash Kumar

Indian Institute of Science Education and Research Kolkata

Tapas Kumar Sengupta ( $\sim$ senguptk@iiserkol.ac.in )

Indian Institute of Science Education and Research-Kolkata https://orcid.org/0000-0001-6482-914X

\section{Original Article}

Keywords: Bacillus thuringiensis, Biofilm, Glucose, Cell-surface hydrophobicity

Posted Date: February 4th, 2021

DOI: https://doi.org/10.21203/rs.3.rs-185959/v1

License: (9) This work is licensed under a Creative Commons Attribution 4.0 International License.

Read Full License

Version of Record: A version of this preprint was published at Current Microbiology on December 14th, 2021. See the published version at https://doi.org/10.1007/s00284-021-02699-z. 


\section{Abstract}

Bacillus thuringiensis are agriculturally and medically important bacteria as they produce insecticidal Cry proteins and can form biofilm on different plant surfaces. Previous studies reported that the ubiquitous carbon source glucose could induce restricted motility and fractal pattern formation in the growing colonies of $\mathrm{pH}$, salt and arsenate tolerant Bacillus thuringiensis KPWP1. As bacteria are evolved with the ability to exhibit multicellular behaviour and biofilm formation under limiting conditions for survival, the present study was focused on exploring the effect of glucose in biofilm formation by Bacillus thuringiensis KPWP1. Significant rise in biofilm loads were observed with increased glucose concentrations in growth media. Compared to control, six times more biofilm load was marked in presence of $2 \%$ of glucose. Interestingly, it was observed that the effect was glucose specific and also not due to any change in sugar induced physicochemical property of the growth media as addition of galactose or arabinose could not induce any significant increase in KPWP1 biofilm load. Scanning electron-, confocal laser scanning-microscopic studies and biochemical tests revealed that increased concentrations of glucose could induce increased production of exopolymeric substances, increased number of densely-packed micro-colonies in KPWP1 biofilm and increased hydrophobicity and adherence properties in KPWP1cells.

\section{Introduction}

Thousands of years ago, human beings adapted a survival strategy to stay in communities, especially communities inclusive of people with one-of-a-kind abilities. They realized that a community is far more likely to continue to exist through the department of hard work- one makes food, other gathers source, nonetheless any other protects the community towards invaders (Johnson LR et al.2008; Verplaetse et al.2015; Verplaetse et al.2017). Microbes exhibit two kinds of growth modes i.e. planktonic cells and sessile aggregate that is called the biofilm. Biofilms are a critical component of the natural surroundings (Jamal et al.2015) and they are found in multiple habitats from soil (Maeder et al.2002) to space (Horneck et al.2006), roots of the tree to the leaves, outer dead skin layer (Grice et al.2009) to the intestines.

Majority of microorganisms have the potency to construct biofilm on a wide range of surfaces including biotic and abiotic surfaces (Verplaetse et al.2015) by producing the extracellular polymeric substance (EPS) (Jamal et al.2015; Verplaetse et al.2015). For the most part in biofilm, microorganisms involve about $10 \%$ of the dry mass, whereas the remaining $90 \%$ are possessed by EPS (Kavitaet al. 2013). It mainly consists of different macromolecules like proteins, polysaccharides, lipids and extracellular DNA (Kavita et al.2014). The EPS fill in between the shape of the biofilm constituents.

Numerous factors, including source and abundance of nutrients, osmolality, temperature, and anaerobiosis were suggested to affect biofilm formation and EPS production (Lim et al. 2004). Although glucose is a preferred carbon source for most of the living organisms, there are conflicting reports on the effect of glucose in bacterial biofilm formation. Although it was observed that glucose can inhibit biofilm 
formation by multiple species of Enterobacteriaceae, it was reported that glucose supplementation in growth media enhance biofilm formation in E. faecalis. Such divergent results indicate a bimodal fashion in occurrence of glucose mediated biofilm formation in enterococci (Pillai et al.2004).

Bacteria belonging to Bacillus cereus group display a broad range of existence and ecological niches and consist of useful as well as pathogenic lines. Bacillus thuringiensis, a Gram-positive, spore-forming microbe, belong to Bacillus cereus group which is used as a biological pesticide as this microbe produces insecticidal toxins (cryl, cryIV, crylll etc) that target selected insect hosts ( Gill et al.1992;Bravo et al.2007; Gill et al.1992; Guan et al. 2014;Qi JiaHeLing et al. 2016). Initially, spores and crystalline insecticidal proteins produced by $B$. thuringiensis have been used to control insect pests since the 1950s and are often applied as liquid sprays (Saiyad et al.2017). But the problems arise when they get washed off from the plant surface due to heavy rainfall and irrigation. If biofilm formation of $B$. thuringiensis is encouraged onto the plant surface, the cells can adhere to the plant surface by secreting an extracellular matrix and ensure the insecticidal property without being engineered the crop. Therefore, studies on biofilm formation by Bacillus thuringiensis are increasingly recognized as an important area of research. Previous studies from our laboratory revealed that increased concentration of glucose in growth media resulted in decreased number of flagella on KPWP1 cell surface (Roy et al.2010). There are contrasting reports on the relation between swarming and biofilm formation in bacterial world. In one hand, it is generally observed that swarming motility and biofilm formation are inversely correlated (Caiazza et al.2007; Verstraeten et al.2008; Guttenplan et al.2013) whereas, recent findings indicate that for some bacteria, there may be a positive correlation between bacterial swarming and biofilm formation (FuenteNúñez et al.2012; Park et al.2018). It is, therefore, important to know whether swarming and biofilm formation are inversely or positively correlated in Bacillus thuringiensis KPWP1 and also to know whether glucose can influence the biofilm formation by Bacillus thuringiensis KPWP1.

The aim of the present study was to investigate the effect of glucose in biofilm formation of Bacillus thuringiensis KPWP1 and also to characterize the changes in EPS composition, cell morphology and surface hydrophobicity in KPWP1 biofilms due to the presence of differential concentrations of glucose in growth media.

\section{Materials And Methods}

\subsection{Bacterial strains and growth conditions}

Bacillus thuringiensis KPWP1, (Roy et al.2010) isolated from Kolkata port water, were grown in 24-well plates in nutrient broth (peptone $-0.5 \%, \mathrm{NaCl}-0.5 \%$ and beef extract- $0.3 \%$ ) in presence of different concentration of glucose, arabinose and galactose as required for experiments. Planktonic growth of bacteria was monitored by measuring the turbidity/O. D of bacterial suspension at $600 \mathrm{~nm}$ by using a UVvis spectrophotometer (Schimadzu UV 2600)

\subsection{Biofilm assay}


Biofilm formation was assayed was performed by the method described by (Pui et al.2017) with minor modification. For assay of biofilm formation by Bacillus thuringiensis, $10^{6}$ cells number of KPWP1 cells in $1 \mathrm{ml}$ were grown for 48 hours in nutrient broth in 24 well plates $\mathrm{n}$ presence of $1 \%$ and $2 \%$ of sugars at $37^{\circ} \mathrm{C}$ under shaking condition (150 rpm). The planktonic cells were removed and the growth of planktonic cells was measured spectrophotometrically. Wells (contained biofilm) of plates were gently washed with phosphate buffered saline (PBS) twice and incubated with $0.1 \%$ crystal violet (CV) for 15 mins at room temperature. After incubation, excess stain was removed by washing the wells with PBS. $\mathrm{CV}$ attached to the biofilm was extracted with $1 \mathrm{ml}$ of $30 \%$ acetic acid. The biofilm load was calculated by measuring the optical density (O.D) of the extracted crystal violet at $600 \mathrm{~nm}$ by using microplate.

\subsection{Confocal laser scanning microscopy of biofilm}

Confocal laser scanning microscopy (CLSM) was performed to measure three-dimensional structure and related topological parameters of KPWP1 biofilms. For that, KPWP1 biofilms were formed on coverslip by using the same method as described above (method Sect. 2.3). The coverslips, containing the biofilms, were washed with PBS two times gently and then stained with $0.001 \%$ acridine orange and incubated in dark for 10 mins. The stained coverslips were washed with PBS to remove the excess stain and observed under confocal microscope

The bio-volume ( $\mu \mathrm{m} 3 / \mu \mathrm{m} 2)$, mean thickness $(\mu \mathrm{m})$, volume ( $\mu \mathrm{m} 3)$, skewness and kurtosis of KPWP1 were quantified from the confocal stacks using the Zen software. The bio-volume is defined as the volume of the biomass $(\mu \mathrm{m} 3)$ divided by the surface area of the substratum $(\mu \mathrm{m} 2)$. The skewness determines the porosity in the biofilm which helps the cells to get access to the nutrient. The kurtosis value determines the adherence property of the bacteria (Dasgupta et al.2013).

\subsection{Scanning Electron Microscopy}

In order to visualize the biofilm formed by KPWP1 cells, SEM was performed by using the protocol described by Chakraborty et al.2016 with minor modification.

Biofilm was first formed onto the coverslip placed vertically in 24 well plates. The coverslips were washed with PBS and fixed with $200 \mu$ of glutaraldehyde (2.5\%) solution for 1 hour at room temperature in dark. The coverslips were then re-suspended in $200 \mu \mathrm{l}$ of $0.1 \%$ OsO4 and incubated at room temperature for 30 minutes. After repeated (three times) wash with PBS, cells were again washed in $30 \%, 50 \%, 70 \%, 90 \%$, and $100 \%$ alcohol respectively. Finally, $20 \mu$ of $100 \%$ ethanol was added onto the coverslips. The samples were then dried using desiccators and then were coated with a thin layer of conducting metal, (goldpalladium) in sputter coater (Quorum technologies Itd.) The samples were observed under field emission scanning electron microscope (Carl Zeiss) using smart SEM software

\subsection{Live-dead staining of biofilm}

The presence of live and dead cells in KPWP1 biofilm was measured by staining the biofilm (formed on coverslips) with the live/dead BACLIGHT bacterial viability kit (Invitrogen, paisley, UK) (He et al.2017). The 
images of live and dead cells were stained with SYTO 9 dye and Propidium iodide (PI), respectively and the images were captured by using fluorescence microscope (Olympus provis ax70)

\subsection{Microbial adhesion to the hydrocarbons (MATH) assay}

In order to measure the hydrophobicity of cell surface of bacteria grown under different conditions, MATH assay was performed by using hydrophobic hydrocarbon n-hexadecane (Sigma, purity > 99\%) (Tyfa et al. 2015). For that, KPWP1 cells were grown in Nutrient Broth in presence of different concentrations of glucose. The optical densities of cell suspensions were adjusted to 0.6 by diluting the suspensions with PBS (A0). Next, $1.5 \mathrm{ml}$ of adjusted cell suspensions were thoroughly mixed with $0.5 \mathrm{ml}$ of $\mathrm{n}$-hexadecane for $30 \mathrm{~min}$ at room temperature. The mixtures were then allowed to settle for $30 \mathrm{~min}$ and the aqueous layers were collected and OD of the collected aqueous layers was measured at $600 \mathrm{~nm}(\mathrm{~A} 1)$. The percentage hydrophobicity was calculated by the formula;

Percentage hydrophobicity $=\left[1-\mathrm{A}_{1} / \mathrm{A}_{0}\right] * 100$

\subsection{Staining of Exopolymeric substances (EPS) components with concanavalin A and Nile red}

The EPS is consisting of embedded carbohydrate, proteins and lipids which gives a structural integrity for the three-dimensional biofilm lattice. The dispersion of extracellular polysaccharides was examined utilizing fluorochromes that interact with particular oligosaccharide subunits. Concanavalin $A($ Con $A)$ was used to stain glycoconjugates (mannose specific) part of biofilm flocs. The biofilm onto the coverslips was stained with $500 \mathrm{mg} / \mathrm{l}$ of the dye followed by incubation for 15 mins in dark [35]. The samples were washed with PBS and observed under confocal laser scanning microscopy with an excitation wavelength of $485 \mathrm{~nm}$ and emission at $530 \mathrm{~nm}$. Similarly, Nile red (2.5mg/l) localized lipids such as triglycerides phospholipids, and neutral lipid droplets within cells. The staining procedure was the same as above about the incubation time, in this case, is 10 mins followed by PBS wash (Chen et al.2009).

\subsection{Quantification of carbohydrate, protein and eDNA in EPS of KPWP1 biofilms}

The anthrone method was used for quantification of the carbohydrate content of EPS (Khan et al.2014). In brief, $160 \mu$ l of anthrone reagent $\left(0.125 \%\right.$ anthrone [wt./vol] in $94.5 \%$ [vol/vol] $\left.\mathrm{H}_{2} \mathrm{SO}_{4}\right)$ was mixed with $80 \mu \mathrm{l}$ of the sample and incubated at $100^{\circ} \mathrm{C}$ for $14 \mathrm{~min}$ and after that cooled at $4^{\circ} \mathrm{C}$ for $5 \mathrm{~min}$. The absorbance at $625 \mathrm{~nm}$ was measured utilizing a microplate reader. The Bradford method was used to measure the protein concentration in EPS. In brief, $400 \mu$ of the filtered sample was blended tenderly with $100 \mu \mathrm{l}$ of Bradford reagent 96 well plate. After 5 min of blending, $200 \mu$ l of the blend was utilized to measure the absorbance at $595 \mathrm{~nm}$ with a microplate reader (Kawaguchi et al.2000). For eDNA quantification, KPWP1 biofilms were grown in 24 well plates. The planktonic cells were discarded after 48 hours of incubation and the wells were washed with $0.9 \% \mathrm{NaCl}$ solution for two times. $1 \mathrm{ml}$ of the $0.9 \%$ $\mathrm{NaCl}$ was added to the wells and dispensed properly. The mixture was vortexed vigorously for $1 \mathrm{~min}$ and 
centrifuged at $5,500 \times \mathrm{g}$ at $4^{\circ} \mathrm{C}$ for 10 mins (Panariello et al.2017). The supernatant was collected and the eDNA content was measured by using Nanodrop 1000 spectrophotometer (Thermo scientific Asheville, NC, USA).

\subsection{Statistical analysis}

Graph Prism 6 was used to analyse the data. At least three independent data of anindividual experiment were taken and mean \pm SE was used to express all the results.

\section{Results}

\subsection{Effect of glucose on growth and biofilm formation of Bacillus thuringiensis}

Planktonic cell growth of KPWP1 in presence of glucose was monitored by measuring the turbidity $\left(A_{600}\right)$ by using UV- visible spectrometer. 1.33- and 1.35-fold increase in the growth of the planktonic cells was observed in case of glucose supplemented media with $1 \%$ and $2 \%$ of glucose with respect to control, although, no significant difference in planktonic growth was observed between media containing different glucose percentage (Fig. 1A).

Interestingly, significant rise in biofilm load was observed with the increase in glucose concentrations in growth media. An increment of 11.45 and 18.36 -fold in biofilm load was marked in $1 \%$ and $2 \%$ of glucose supplemented nutrient broth respectively, with respect to control (Nutrient broth without added glucose) (Fig. 1B). The results indicate that KPWP1 was able to form better biofilm in presence of glucose. Moreover, the observed increase in biofilm formation was found to be glucose specific and also not due to any change in physicochemical property (e.g., osmolarity) of growth media as addition of increased concentrations of galactose or arabinose could not induce significant biofilm formation as compared to glucose (Fig. 1C).

\subsection{Topological parameters of KPWP1 biofilm}

In order to characterize the topological parameters of KPWP1 biofilm, confocal laser scanning microscopy was done on KPWP1 biofilm formed on glass coverslips. The bio-volume, mean thickness, biomass, kurtosis and skewness of the 48-hour grown biofilms were analysed to evaluate changes in the biofilm structure with increased glucose concentrations in growth media. As shown in Fig. 2A, 2B and 2C the mean thickness, biovolume and biomass of the bacterial micro-colonies of the 48-h biofilms increased gradually as the glucose concentration increased. The thickness of the biofilm in $1 \%$ and $2 \%$ glucose supplemented media was found to be 1.43 and 1.86 times more than the control while the volume increases by 1.48 and 1.82-folds, respectively (Fig. 2A). The biomass of the KPWP1 biofilm was also observed to be increased by 2.06 -fold in $2 \%$ glucose concentrations (Fig. 2C).

The skewness and kurtosis value are the two statistical parameters that measures porosity in biofilm and adherence of the bacteria to the surface respectively. Lesser the skewness value more is the porosity that 
enables the bacteria to access more nutrient. From the figure (Fig. 2D), the lowest skewness value was observed in the case of $2 \%$ glucose containing media which implies the presence of more porosity in the formed biofilm. The highest kurtosis value (Fig. 2E) was again noticed in $2 \%$ glucose supplemented media states that the adherence property of bacteria increases as glucose is added in the media.

Figure 2F shows representative CLSM images of KPWP1 biofilms, in which the bacterial micro-colonies at in $1 \%$ and $2 \%$ of glucose-containing media were denser, and more aggregated than that of control.

\subsection{Scanning Electron Microscopy of KPWP1 biofilm}

We further explored the effect of glucose on Bacillus thuringeinsis KPWP1 biofilm at the levels of cell morphology and presence of EPS by scanning electron microscopy (SEM). Biofilms were formed on glass coverslips in 24-well microtiter plates and visualised by SEM. The scanning electron microscopy images of KPWP1 cells in biofilms grown in absence of any added glucose revealed that monolayer of a few spores adhering to the coverslips (Fig. 3A.i and 3A.ii). Interestingly, the outcome due to the addition of $1 \%$ glucose in growth media presents a contrasting picture. At the lower magnification, it was detected that heterogenous cell population of KPWP1 adhering to the substratum forming multiple layers with void spaces (Fig. 3B.i. and 3B.ii.) which most probably help in nutrient circulation and excretion of the waste products. The higher magnification of the images revealed that the heterogenous cell population consist of densely packed region of elongated cells, normal healthy cells and spores embedded in EPS of KPWP1 biofilm. The effect of $2 \%$ glucose on KPWP1 biofilm is also captivating. The images revealed the presence of densely packed 3-D structure of KPWP1 cells along with the void spaces with more EPS compare to the $1 \%$ glucose and the higher magnification gives an exact view of the cell morphology where it is observed that the heterogeneously cells population is present trapped in EPS (Fig. 3C.i and 3C.ii.)

\subsection{Glucose induced change in cell surface hydrophobicity in KPWP1 cells}

Cell surface hydrophobicity (CSH) was assessed using the microbial adhesion assay for hydrocarbons (MATH). High CSH enables microorganisms to attach to the hydrophobic surface and form a biofilm. The results show that the least percentage hydrophobicity in control. The maximum percentage hydrophobicity is observed in case $2 \%$ glucose (Fig. 4). The CSH changes as the cell surface macromolecules changes with response to environmental stimulus and nutrient variation. The results clearly depict that addition of $1 \%$ and $2 \%$ glucose in nutrient media increases the percentage hydrophobicity by 3.77 and 4.85 folds respectively. Cell surface hydrophobicity has been distinguished as a measurable trademark for microscopic organisms. This property has been usually investigated in the adherence of microscopic organisms to surfaces. Nutrients play an important role in altering the cell surface hydrophobicity which eventually assists the bacteria to form biofilms. Thus, glucose treatment applied in the present study caused significant variation in biofilm organization. 


\subsection{Live dead staining of bacteria in KPWP1 biofilms}

Both live and dead bacteria embrace the biofilm of Bacillus thuringiensis were observed when they were allowed to develop on $18 \mathrm{~mm}$ coverslips in 24 well plates. By using the BACLIGHT live/dead viability probe (molecular probes), we observed that cell death is manifested, in the micro colonies (Fig. 5). As it is reported that dead cells play important role in the adherence of the bacteria while forming biofilm (Desai et al.2019), imaging of live/dead cells in KPWP1 biofilm, grown in absence and presence of added glucose was performed. The overlapped images of SYTO 9 and PI of the live dead staining depicts that the dead cells (indicate by red) are present at the bottom while the live cells (indicate by green) are present at the top of the biofilm. It is also observed that with the increase of the glucose percentages, the intensity of the dead cells also increases in the KPWP1 biofilm which indicates that the dead cells might helped the live cells to adhere to the substratum during biofilm formation.

Cell death inside microcolonies is an imperative physiological occasion that plays a part in subsequent differentiation and dispersal of a subpopulation of surviving biofilm cells (Webb et al.2003).

\subsection{Identification and estimation of EPS in KPWP1 Biofilm}

Exopolymeric substances in bacterial biofilm are generally made of polysaccharides, proteins, glycolipids and eDNA.

Total polysaccharide was determined biochemically by utilizing anthrone reagent. The carbohydrate content is 8.45 and 31.70 -fold more in $1 \%$ and $2 \%$ glucose (Fig. $6 \mathrm{~A}$ ). Bradford reagent is used to quantify the protein content in the EPS matrix. The results suggest that the total protein content is 9.8 and 36.24fold more in more in $1 \%$ and $2 \%$ glucose with respect to control (Fig. 6B). eDNA was quantified by spectrophotometric analysis. Notably, eDNA contents in KPWP1 biofilm was found to be 1.9 and 5.01 folds more in $1 \%$ and $2 \%$ glucose with respect to the control condition with no added glucose in growth medium (Fig. 6C).

In order to check the relative presence and quantify the carbohydrate residue the EPS matrix was stained with Con A Fig. (6D). The observed intensity for Con A which binds with the mannose residue is 2.8 and 3.1 folds (Fig. $6 \mathrm{E}$ ) more in the matrix $1 \%$ and $2 \%$ glucose with respect to control.

Nile red was used for the assessment of lipids residue present in the EPS matrix of KPWP1. The lipophilic stain Nile red shows the presence of lipid or exceedingly hydrophobic areas within biofilms (Fig. 6F). The intensity for Nile red was 1.74 and 4.60 times more in the EPS matrix of $1 \%$ and $2 \%$ glucose with respect to control (Fig. 6G).

\section{Discussion}

Biofilm development is a multiplex procedure that is influenced by many factors, which include growth condition, stress and surface attributes (Kostakioti et al.2013). Microbial biofilms are captivating areas to study because of their wide varieties in nature, importance in infection and use in bioremediation and 
industries. Advances in molecular and biochemical methods have helped in improving our comprehension of biofilm structure and functions (Velmourougane et al.2017). Exploiting naturally occurring- and in-situ biofilm devolvement are promising ways for future advances in agricultural field as they can possibly give different advantages like increase crop production, protection of the economically important plants from insects and improvement of the crop variety (Franklin et al.2015).Investigations uncovered the intricate conditions experienced in biofilms and during infection create extraordinary heterogeneity inside the populace (Bisht et.al 2019).

Regarding the regulation of biofilm formation, it was reported that in case of Bacillus subtilis, glucose repressed biofilm development through the catabolic control protein CcpA (Stanley et al.2003). On the other hand, interesting and contrasting reports showed that addition of glucose with $\mathrm{NaCl}$ in Tryptic Soy broth (TSB) altogether favoured more biofilm formation in Bacillus cereus cells compared to the addition of glucose or glycerol or $\mathrm{NaCl}$ in growth media (Kwon et al.2017). Many studies suggest that the biofilm develops in restricted supplement of nutrients or in the presence of any sort of stress i.e. antibiotic, high salt or a corrosive substance like acid (Khatoon et al.2018). It is also reported that the nutrient accessibility affects adherence, biofilm arrangement and synthesis of EPS (Xiao et al.2017).

Recent studies revealed the biofilm forming abilities the Bacillus thuringiensis isolates invitro and on plant surfaces (Verplaetse et al.2015). B. thuringiensis biofilms on plant surfaces thus can help the plant by protecting it from the pathogens like agricultural pests. It is also reported that Bacillus thuringiensis spores have a higher hydrophobicity, conferring them a higher adhesive potential to diverse materials (Wiencek et al.1990). Interesting findings in recent times revealed that Bacillus thuringiensis cells undergoes differentiations and represent a mixed population of heterogenous cells under stressed conditions (Verplaetse et al.2015).

Previous study from our laboratory revealed that addition of higher concentrations of glucose in growth media could induce restricted swarming motility due to impaired flagellation in Bacillus thuringiensis KPWP1 on nutrient agar plates (Roy et al.2010). This finding had raised the question whether the restricted motility in presence of glucose can trigger the biofilm forming ability in Bacillus thuringiensis KPWP1 cells? The present study was therefore to explore whether restricted motility induced by glucose can regulate the adherence property, production of EPS components, thus biofilm formation ability in Bacillus thuringiensis KPWP1. The results from this present study clearly indicate that the presence of added glucose in nutrient-rich media induces biofilm formation (Fig. 1B) by KPWP1 as a result of increase in EPS production (Fig. 6) and cell surface hydrophobicity (Fig. 4) and such induced biofilm formation is glucose specific as addition of galactose or arabinose in growth media could not induce any significant biofilm formation by KPWP1(Fig. 1D).

The increased biofilm formation, in presence of glucose, was manifested by the increased thickness (Fig. 2A), bio-volume (Fig. 2B), and biomass (Fig. 2C). Moreover, it was observed that the skewness (Fig. 2D) which reflects the intracellular void spaces and the kurtosis values (Fig. 2E) which expresses the 
adherence of microscopic organisms in KPWP1 biofilm also increased with the increased glucose concentrations in growth media.

It is reported that EPSs add to the mass and 3-D structure of the biofilm framework [46]. The present study revealed that the EPS components i.e. polysaccharides, proteins, lipids and eDNA concentrations increased (Fig. 6A, 6B and 6C) in KPWP1 biofilms with the increase in glucose concentration in growth media. In the 48-h grown biofilms, EPS contents - sugar, proteins lipid and eDNA were altogether higher in the presence of higher concentrations of glucose than that of control condition and supports the observed increase in physical appearance of EPS in SEM images of KPWP1 biofilms grown in different glucose concentrations (Fig. 3). Furthermore, it is also indicative that the EPSs formed in presence of 1 and $2 \%$ glucose helps in the adherence of the bacteria to the surface (Fig. 6). Collectively, the present study uncovered the effect of glucose on biofilm arrangement of KPWP1 cells. Glucose prompts the adherence property of the Bacillus thuringiensis KPWP1 cells and induces more EPS production resulting in more biofilm formation.

The results of the present study signify that restricted motility in Bacillus thuringiensis KPWP1 induced by glucose helps the bacterial cells to form biofilm, thus give a major premise to a more elaborate investigation of the in-vivo biofilm formation by this insecticidal bacterium Bacillus thuringiensis on plant surfaces, particularly in response to glucose.

\section{Declarations}

\section{Conflicts of interest/Competing interests}

The authors of the paper declare that there is no conflict of interest.

\section{Ethics approval:}

Not required

\section{Consent for publication:}

All authors have consent for publication of this study

\section{Funding:}

The study was funded by IISER Kolkata.

\section{Authors' contributions:}


SJ and TKS conceived the study and designed the experiments. SJ, NB and AB performed the experiments and analysed the data. SJ and TKS have written the manuscript.

\section{Acknowledgements}

The authors gratefully acknowledge Mr Ritabrata Ghosh for help with confocal imaging and Mr Kashinath Sahu for help with SEM imaging at IISER-K. SJ, NB and AB are recipients of fellowship from IISER-K. TKS acknowledge the funding received from IISERK.

\section{References}

1. Beauregard PB, Chai Y et al (2013) Bacillus subtilis biofilm induction by plant polysaccharides. Proc Natl Acad Sci 110:E1621-E1630

2. Bisht K, Wakeman CA et al (2019) Discovery and Therapeutic Targeting of Differentiated Biofilm Subpopulations. Front Microbiol 10:1908

3. Bravo A, Gill SS et al (2007) Mode of action of Bacillus thuringiensis Cry and Cyt toxins and their potential for insect control. Toxicon Off J Int Soc Toxinology 49:423-435

4. Caiazza NC, Merritt JH et al (2007) Inverse regulation of biofilm formation and swarming motility by Pseudomonas aeruginosa PA14. J Bacteriol 189(9):3603-3612

5. Chakraborty B, Mallick A et al (2016) Deciphering a survival strategy during the interspecific competition between Bacillus cereus MSM-S1 and Pseudomonas sp. MSM-M1. R Soc Open Sci 3(11):160438

6. Chen W, Zhang C et al (2009) A high throughput Nile red method for quantitative measurement of neutral lipids in microalgae. J Microbiol Methods 77(1):41-47

7. Costerton JW (1999) Introduction to biofilm. Int J Antimicrob Agents 11:217-221

8. Dasgupta D, Ghosh R et al (2013) Biofilm-Mediated Enhanced Crude Oil Degradation by Newly Isolated Pseudomonas Species. ISRN Biotechnology. 10.5402/2013/250749

9. de la Fuente-Núñez C, Korolik V et al (2012) Inhibition of bacterial biofilm formation and swarming motility by a small synthetic cationic peptide. Antimicrob Agents Chemother 56(5):2696-2704

10. Desai S, Sanghrajka K et al (2019) High Adhesion and Increased Cell Death Contribute to Strong Biofilm Formation in Klebsiella pneumoniae. Pathogens 8(4):277

11. Franklin MJ, Chang $C$ et al (2015) New Technologies for Studying Biofilms. Microbiol Spectr 3(4):10

12. Gill SS, Cowles EA et al (1992) The mode of action of Bacillus thuringiensis endotoxins. Annu Rev Enlamal 37:615-636

13. Grice EA et al (2009) Topographical and Temporal Diversity of the Human Skin Microbiome. Science 324:1190-1192

14. Guan P, Dai X et al (2014) Bacillus thuringiensis subsp. sichuansis strain MC28 produces a novel crystal protein with activity against Culex quinquefasciatus larvae. World Journal of Microbiology 
Biotechnology 30:1417-1421

15. Guttenplan SB, Kearns DB et al (2013) Regulation of flagellar motility during biofilm formation. FEMS Microbiol Rev 37(6):849-871

16. He S, Hong X et al (2017) Rapid quantification of live/dead lactic acid bacteria in probiotic products using high-sensitivity flow cytometry. Methods Appl Fluoresc 5:024002

17. Horneck G (2006) Bacterial Spores Survive Simulated Meteorite Impact. Biological Processes Associated with Impact Events. 41-53

18. Jahid IK, Lee NY et al (2013) Influence of glucose concentrations on biofilm formation, motility, exoprotease production, and quorum sensing in Aeromonas hydrophila. J Food Prot 76:239-247

19. Jamal M, Tasneem U et al (2015) Bacterial Biofilm: Its Composition, Formation and Role in Human Infections. Res Rev J Microbiol Biotechnol 4:3

20. Johnson LR (2008) Microcolony and biofilm formation as a survival strategy for bacteria. J Theor Biol 251:24-34

21. Kavita K, Mishra A et al (2013) Extracellular polymeric substances from two biofilm forming Vibrio species: Characterization and applications. Carbohydr Polym 94:882-888

22. Kavita K, Singh VK et al (2014) Characterisation and anti-biofilm activity of extracellular polymeric substances from Oceanobacillus iheyensis. Carbohydr Polym 101:29-35

23. Kawaguchi T, Decho AW et al (2000) Biochemical Characterization of Cyanobacterial Extracellular Polymers (EPS) from Modern Marine Stromatolites (Bahamas). Prep Biochem Biotechnol 30:321330

24. Khan AH, Minhas NM et al (2014) Estimation of carbohydrate, starch, protein and oil contents of maize (Zea mays). Eu J Aca Res II:5230-5240

25. Khatoon Z, McTiernan CD et al (2018) Bacterial biofilm formation on implantable devices and approaches to its treatment and prevention. Heliyon 4(12):e01067

26. Kostakioti M, Hadjifrangiskou M et al (2013) Bacterial biofilms: development, dispersal, and therapeutic strategies in the dawn of the postantibiotic era. Cold Spring Harb Perspect Med 3(4):a010306

27. Kwon M, Hussain MS et al (2017) Biofilm formation of Bacillus cereus under food-processing-related conditions. Food Sci Biotechnol 26(4):1103-1111

28. Lim Y, Jana M et al (2004) Control of Glucose- and NaCl-Induced Biofilm Formation by rbf in Staphylococcus aureus. J Bacteriol 186:722-729

29. Lu Y, Wu K et al (2012) Widespread adoption of Bt cotton and insecticide decrease promotes biocontrol services. Nature 487:362

30. Maeder P, Fliessbach A et al (2002) Soil Fertility and Biodiversity in Organic Farming. Science 296:1694-1697

31. Majed R, Faille C et al (2016) Bacillus cereus Biofilms-Same, Only Different. Front Microbiol 7:1054 
32. Panariello BHD, Klein MI et al (2017) Inactivation of genes TEC1 and EFG1 in Candida albicans influences extracellular matrix composition and biofilm morphology. J Oral Microbiol 9(1):1385372

33. Park K, Mok J et al (2018) Food-borne outbreaks, distributions, virulence, and antibiotic resistance profiles of Vibrio parahaemolyticus in Korea from 2003 to 2016: a review. Fish Aquat Sci 21:3

34. Pillai SK, Sakoulas G et al (2004) Effects of Glucose on fsr-Mediated Biofilm Formation in Enterococcus faecalis. J Infect Dis 190:967-970

35. Pui CF, Apun K et al (2017) Microtitre Plate Assay for the Quantification of Biofilm Formation by Pathogenic Leptospira. Research Journal of Microbiology 12:146-153

36. Qi JiaHeLing, Takahashi $\mathrm{N}$ et al (2016) A potential of biofilm formation by entomopathogenic Bacillus thuringiensis on tomato root surface. Int J Trop Agric 34:369-375

37. Roy MK, Banerjee P et al (2010) Glucose induced fractal colony pattern of Bacillus thuringiensis. J Theor Biol 265:389-395

38. Saiyad S (2017) Application of Bacillus thuringiensis as an effective tool for insect pest control. IOSR Journal of Agriculture Veterinary Science 10:27-29

39. Salama Y, Chennaoui M et al (2016) Characterization, structure, and function of extracellular polymeric substances (EPS) of microbial biofilm in biological wastewater treatment systems: a review. Desalination Water Treat 57:16220-16237

40. Schnepf E, Crickmore N et al (1998) Bacillus thuringiensis and Its Pesticidal Crystal Proteins. Microbiol Mol Biol Rev 62:3775-3806

41. Stanley NR, Britton RA et al (2003) Identification of catabolite repression as a physiological regulator of biofilm formation by Bacillus subtilis by use of DNA microarrays. J Bacteriol 185(6):1951-1957

42. Strathmann M, Wingender $\mathrm{J}$ et al (2002) Application of fluorescently labelled lectins for the visualization and biochemical characterization of polysaccharides in biofilms of Pseudomonas aeruginosa. J Microbiol Methods 50(3):237-248

43. Tyfa A, Kunicka-Styczyńska A et al (2015) Evaluation of hydrophobicity and quantitative analysis of biofilm formation by Alicyclobacillus sp. Acta Biochim Pol 4:785-790

44. Velmourougane K, Prasanna R et al (2017) Agriculturally important microbial biofilms: Present status and future prospects. J Basic Microbiol 57(7):548-573

45. Verplaetse E, Slamti L et al (2017) Two distinct pathways lead Bacillus thuringiensis to commit to sporulation in biofilm. Res Microbiol 168:388-393

46. Verplaetse E, Slamti L et al (2015) Cell Differentiation in a Bacillus thuringiensis Population during Planktonic Growth, Biofilm Formation, and Host Infection. mbio 6:e00138-e00115

47. Verstraeten N, Braeken $\mathrm{K}$ et al (2008) Living on a surface: swarming and biofilm formation. Trends in microbiology 16:496-506

48. Webb, Jeremy S et al (2003) Cell death in Pseudomonas aeruginosa biofilm development. J Bacteriol 15:4585-4592 
49. Wiencek K, Klapes A et al (1990) Hydrophobicity of Bacillus and Clostridium spores. Appl Environ Microbiol 56(9):2600-2605

50. Xiao J, Hara A et al (2017) Biofilm three-dimensional architecture influences in situ pH distribution pattern on the human enamel surface. Int J Oral Sci 9:74-79

51. Yaron S, Römling U et al (2014) Biofilm formation by enteric pathogens and its role in plant colonization and persistence. Microbial biotechnology 7:6: 496-516

\section{Figures}

A

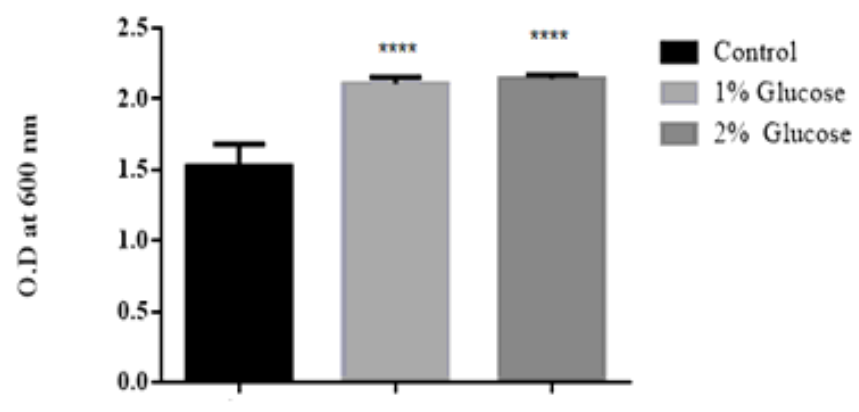

C

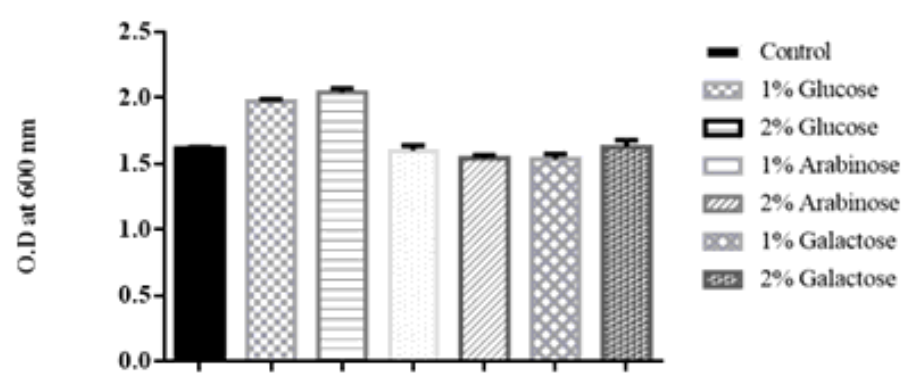

B

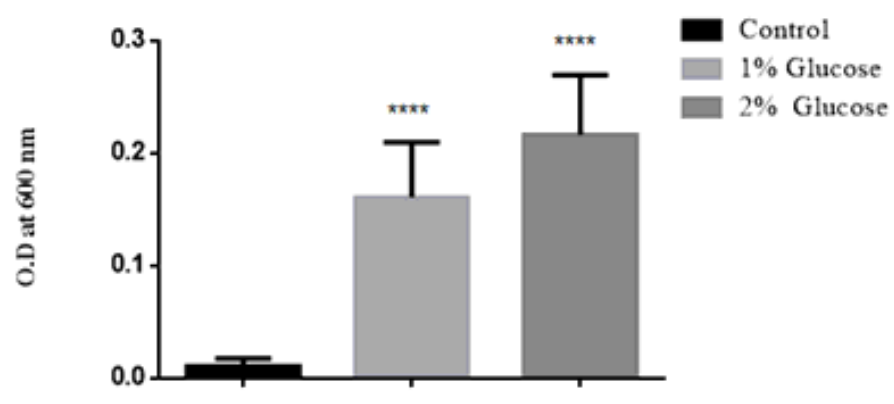

D

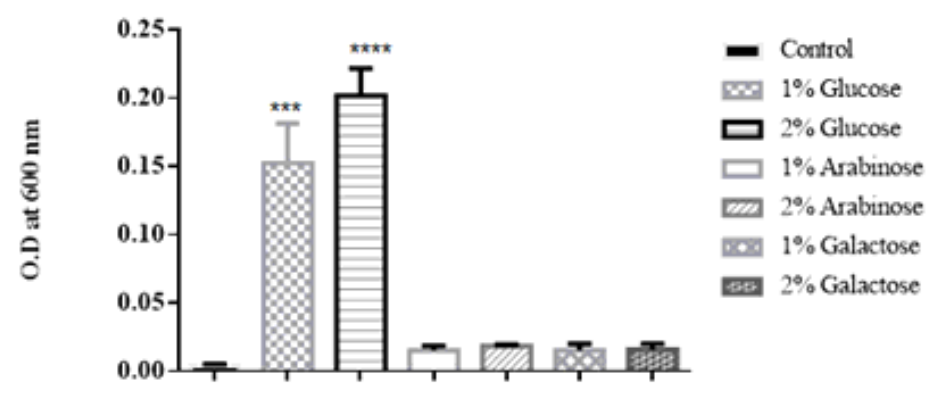

Figure 1

Growth and biofilm formation by Bacillus thuringiensis KPWP1 in absence and presence of glucose. Planktonic growth of KPWP1 in NB after 48 hours of incubation in absence and presence glucose (A) and other sugars (C). Biofilm formation of KPWP1 in NB after 48 hours of incubation in absence and presence of glucose (B) and other sugars (D). Data represent mean values $\pm S E$ of 3 biological replicates. The experiment was repeated three times. Asterisks indicate statistically significant differences when compared to control $\left({ }^{*} \mathrm{P}<0.05 ;{ }^{\star *} \mathrm{P}<0.01 ;{ }^{* \star *} \mathrm{P}<0.001\right.$; two-tailed Student's-test). 
A

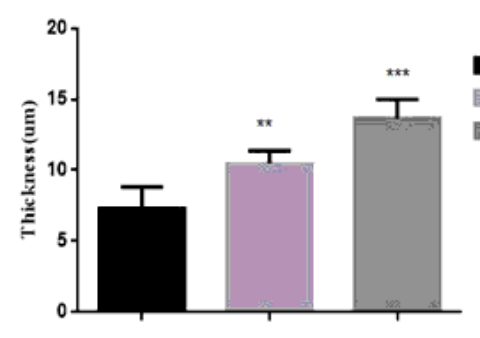

C

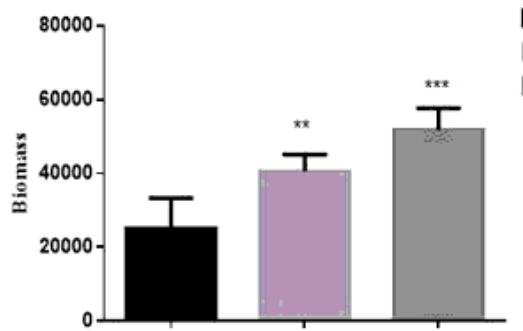

E

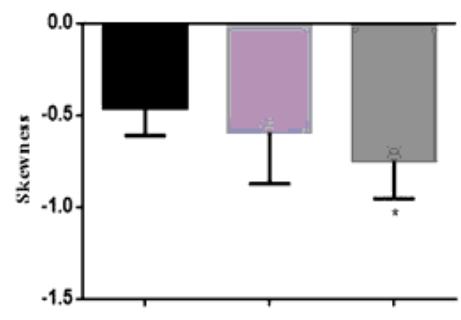

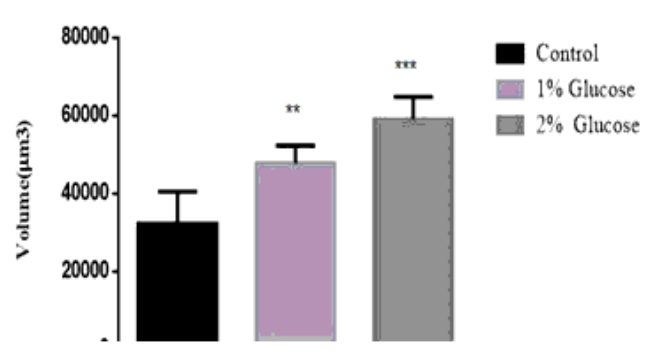

$\mathrm{D}$
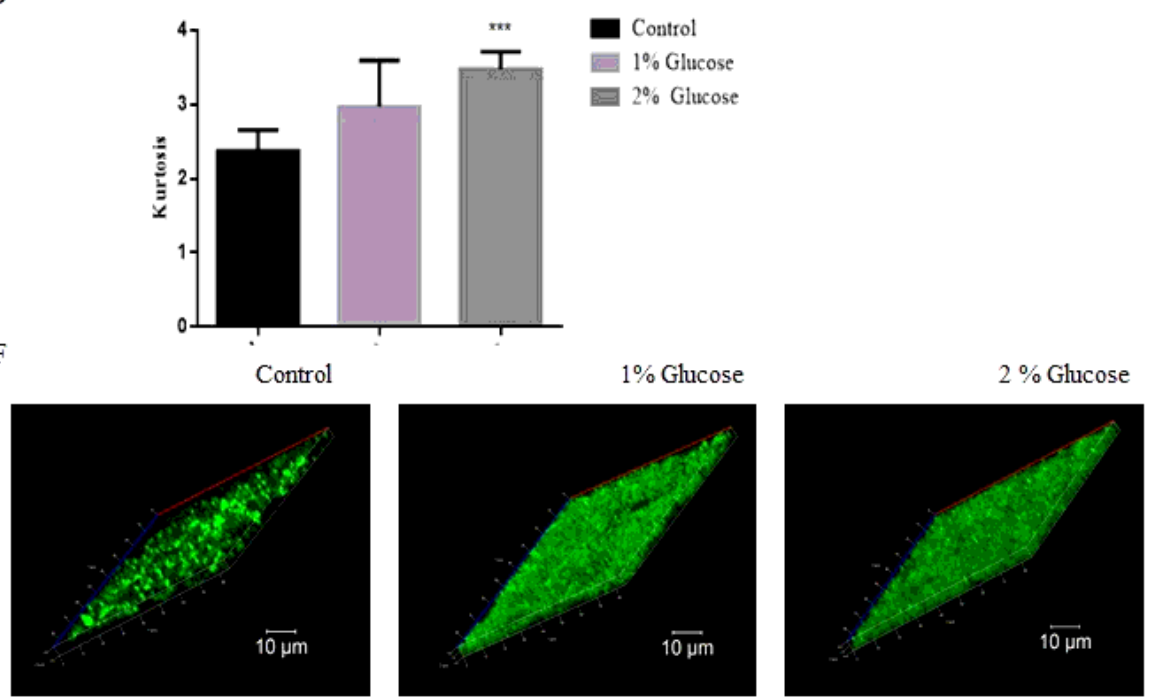

Figure 2

Topological feature of the biofilm formed by Bacillus thuringiensis KPWP1 in 1\% Glucose and $2 \%$ Glucose after 48 hours of incubation,thickness (A) volume, (B) biomass (C), kurtosis (D), skewness (E) and representative CLSM images of biofilm grown in presence of different percentages of glucose of KPWP1 (F). Data represent mean values \pm SE of 3 biological replicates. The experiment was repeated three times. Asterisks indicate statistically significant differences when compared to control $\left({ }^{*} P<0.05\right.$; ${ }^{\star \star} \mathrm{P}<0.01 ; * \star \star P<0.001$; two-tailed Student's-test). 
A.i

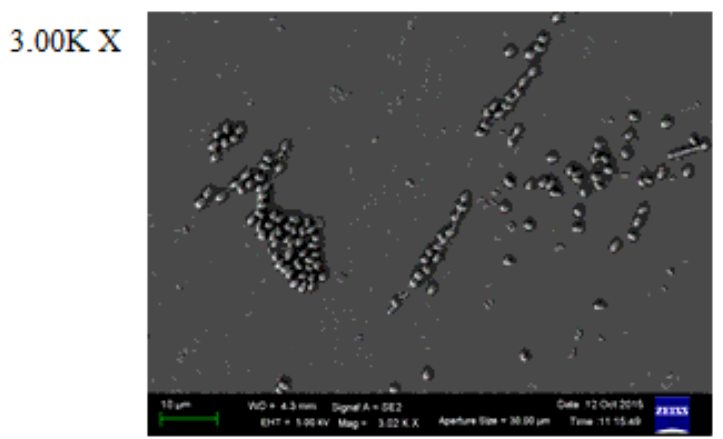

A.ii

$10.00 \mathrm{~K} \mathrm{X}$

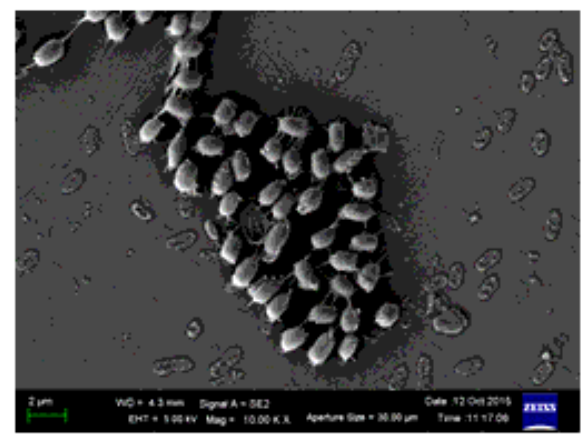

B.i

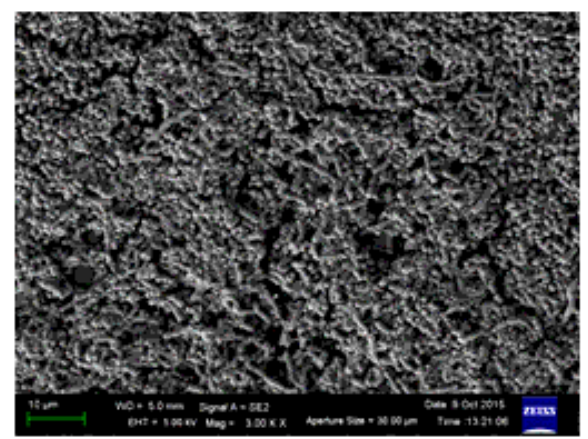

B.ii

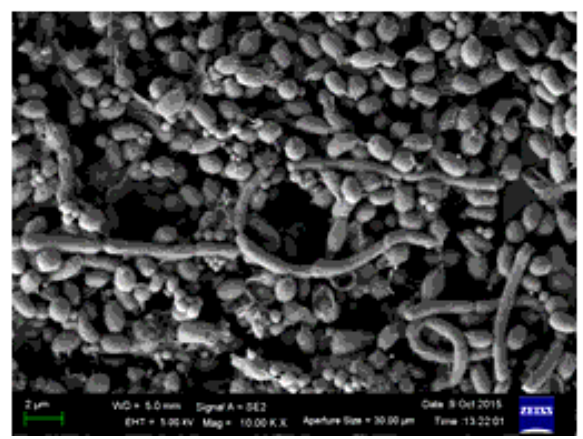

C.i $2 \%$ Glucose

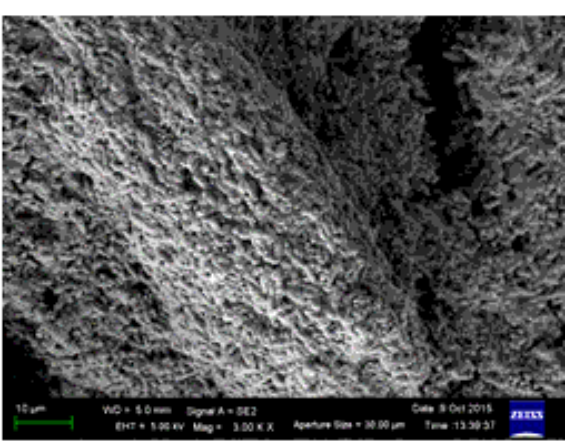

C.ii

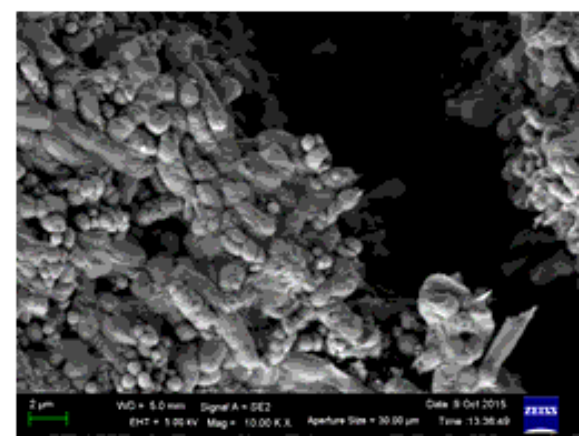

\section{Figure 3}

Scanning Electron Micrograph (SEM) of biofilm of KPWP1 that developed on a glass coverslip after 48 hours period in nutrient broth with different percentage of glucose, Control (no Glucose added) at 3.00K X and $10.00 \mathrm{~K} X$ magnification (A.i, A.ii), $1 \%$ Glucose at 3.00K X and 10.00K X magnification (B.i, B.ii), $2 \%$ Glucose at $3.00 \mathrm{~K} X$ and $10.00 \mathrm{~K} X$ magnification (C.i, C.ii) respectively.

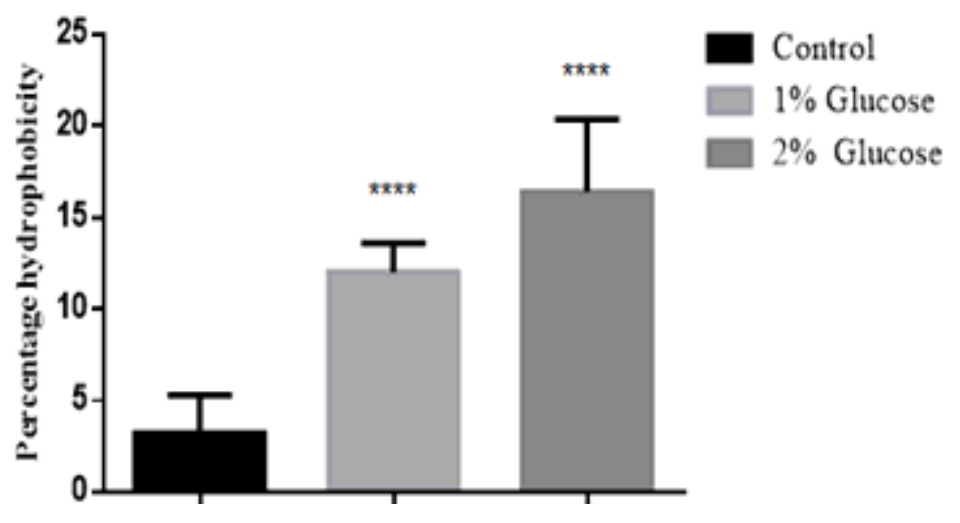

Figure 4

Estimation of adhesion property of KPWP1 cells grown in absence and presence of glucose. 


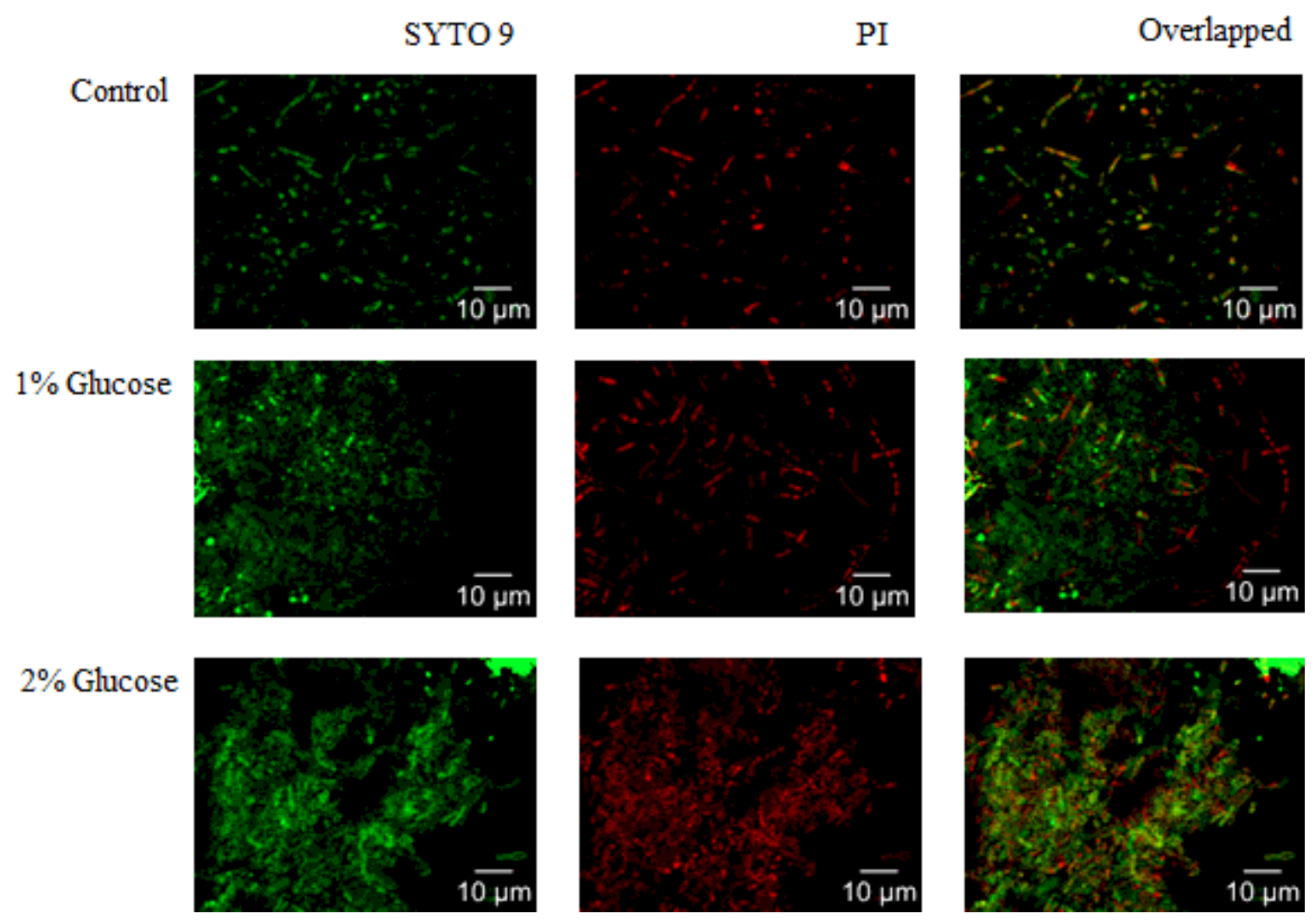

Figure 5

Analysis of Live /dead cells in the biofilm of KPWP1 by using PI and Syto 9. 
A

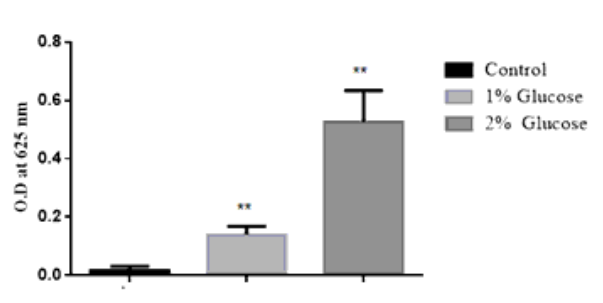

D

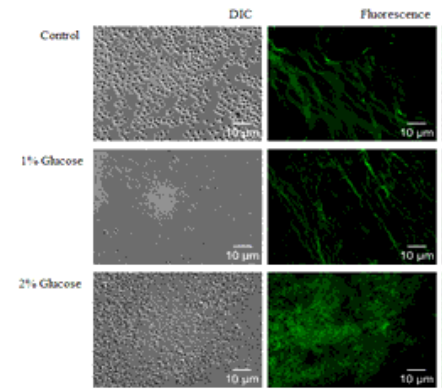

$\mathbf{F}$

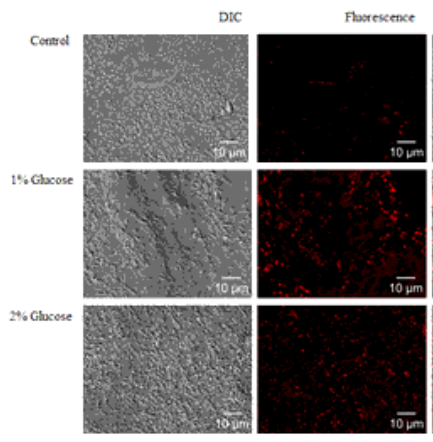

Overappes

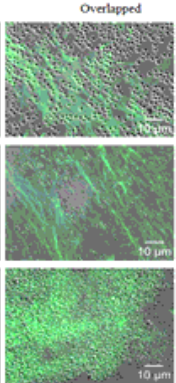

Overibped

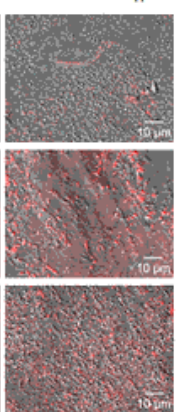

B

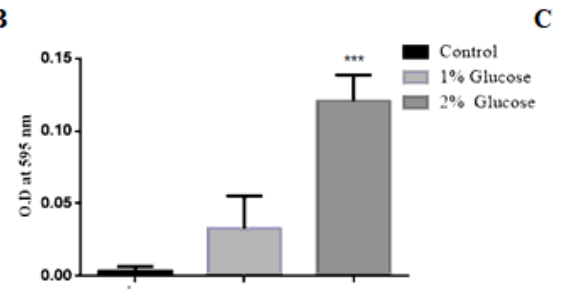

C

$\mathbf{E}$

G
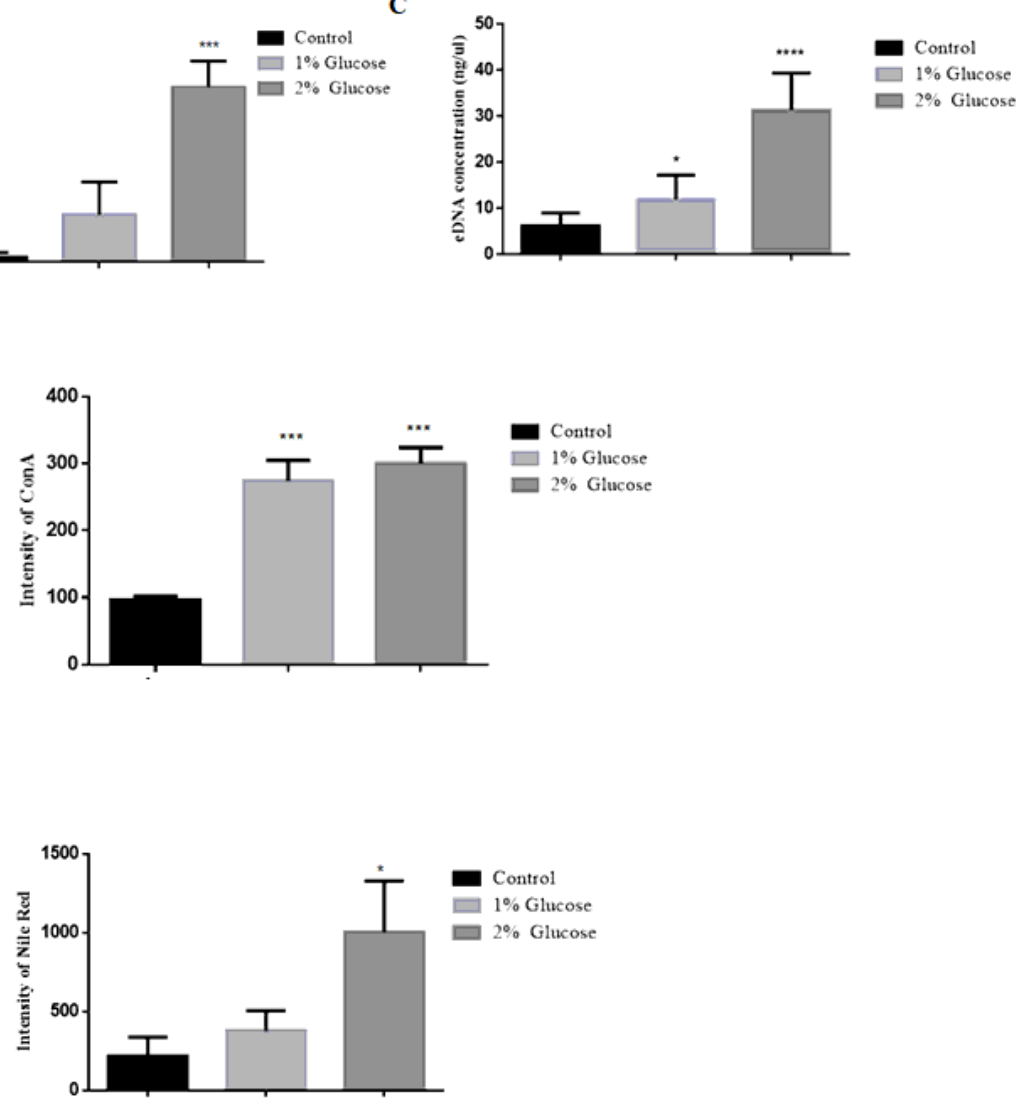

Figure 6

Quantification of Exo polymeric substance in KPWP1 biofilm in absence and presence of Glucose.

Carbohydrate residue (A), Protein (B), eDNA (C), Staining and quantification of mannose respectively by Con $A(D, E)$ and Staining of lipid by quantification of respectively by Nile Red. Data represent mean values $\pm S E$ of 3 biological replicates. The experiment was repeated three times. Asterisks indicate statistically significant differences when compared to control $\left({ }^{\star} P<0.05 ;{ }^{*} P<0.01 ;{ }^{\star \star \star *} P<0.001\right.$; two-tailed Student's-test). 